\title{
A Controlled Human Infection Model of Group A Streptococcus Pharyngitis: Which Strain and Why?
}

Joshua Osowicki a,b,c

Kristy I. Azzopardi a

Liam McIntyre $^{\mathrm{d}}$

Tania Rivera-Hernandez ${ }^{\mathrm{e}}$

Cheryl-lynn Y. Ong e

Ciara Baker ${ }^{a}$

Christine M. Gillen e

Mark J. Walker ${ }^{\mathrm{e}}$

Pierre R. Smeesters a,b,f,g

Mark R. Davies ${ }^{\mathrm{d}}$

Andrew C. Steer ${ }^{\text {a,b,c }}$
a. Tropical Diseases, Murdoch Children's Research Institute, Melbourne, Victoria, Australia.
b. Department of Paediatrics, University of Melbourne, Victoria, Australia
c. Infectious Diseases Unit, Department of General Medicine, The Royal Children's Hospital Melbourne, Victoria, Australia.
d. Department of Microbiology and Immunology, Peter Doherty Institute for Infection and Immunity, The University of Melbourne, Victoria, Australia.
e. School of Chemistry and Molecular Biosciences and Australian Infectious Diseases Research Centre, The University of Queensland, St Lucia, Queensland, Australia.
f. Paediatric Department, Academic Children Hospital Queen Fabiola, Université Libre de Bruxelles, Brussels, Belgium
g. Molecular Bacteriology Laboratory, Université Libre de Bruxelles, Brussels, Belgium.

Running title: Group A Streptococcus Human Challenge Strain

Keywords: group A Streptococcus, Streptococcus pyogenes, controlled human infection, human challenge, vaccine development

\section{Corresponding author:}

Dr Joshua Osowicki

joshua.osowicki@rch.org.au

Tropical Diseases

Murdoch Children's Research Institute

50 Flemington Road

Parkville, 3052

Victoria, Australia

\section{Alternate corresponding author:}

Professor Andrew C Steer

andrew.steer@rch.org.au

Tropical Diseases

Murdoch Children's Research Institute

50 Flemington Road

Parkville, 3052

Victoria, Australia 


\section{Abstract}

Background: Group A Streptococcus (GAS) is a major cause of global infection-related morbidity and mortality. A modern controlled human infection model (CHIM) of GAS pharyngitis can accelerate vaccine development and pathogenesis research. A robust rationale for strain selection is central to meeting ethical, scientific, and regulatory requirements.

Methods: Multi-faceted characterisation studies were done to compare a preferred candidate emm75 (M75) GAS strain against three other strains: an alternative candidate emm12 (M12) strain; an M1 strain used in 1970s pharyngitis CHIM studies (SS-496); and a representative (5448) of the globally-disseminated M1T1 clone. A range of approaches were used to explore strain growth, adherence, invasion, delivery characteristics, short- and long-term viability, phylogeny, virulence factors, vaccine antigens, resistance to killing by human neutrophils, and lethality in a murine invasive model.

$\underline{\text { Results: }}$ The strains grew reliably in a medium without animal-derived components, were consistently transferred using a swab method simulating the CHIM protocol, remained viable at $-80^{\circ}$ Celsius, and carry genes for most candidate vaccine antigens. Considering GAS molecular epidemiology, virulence factors, in vitro assays, and results from the murine model, the contemporary strains show a spectrum of virulence with M75 appearing least virulent and 5448 the most. The virulence profile of SS-496, used safely in 1970s CHIM studies, was similar to 5448 in the animal model and virulence gene carriage.

Conclusions: The results of this multi-faceted characterisation confirm the M75 strain as an appropriate choice for initial deployment in the CHIM, with the aim of safely and successfully causing pharyngitis in healthy adult volunteers. 
Importance:

81 Group A Streptococcus (GAS, Streptococcus pyogenes) is a leading global cause of infection-related

82 morbidity and mortality. A modern controlled human infection model (CHIM) of GAS pharyngitis could

83 help to accelerate vaccine development and drive pathogenesis research. Challenge strain selection is

84 critical to the safety and success of any CHIM and especially so for an organism such as GAS with its wide

85 strain diversity and potential to cause severe life-threatening acute infections (e.g. toxic shock syndrome,

86 necrotizing fasciitis) and post-infectious complications (e.g. acute rheumatic fever, rheumatic heart disease,

87 acute post-streptococcal glomerulonephritis). In this paper, we outline the rationale for selecting an emm75

88 strain for initial use in a GAS pharyngitis CHIM in healthy adult volunteers, drawing on the findings of a

89 broad characterization effort spanning molecular epidemiology, in vitro assays, whole genome sequencing,

90 and animal model studies.

91

92 


\section{Introduction}

Group A Streptococcus (GAS, Streptococcus pyogenes) is a major contributor to global infection-related mortality and morbidity. It causes a diverse spectrum of human disease syndromes, from superficial infections (e.g. pharyngitis, impetigo) to invasive disease (e.g. necrotizing fasciitis, toxic shock syndrome) and autoimmune complications (acute rheumatic fever, rheumatic heart disease, glomerulonephritis)(1).

Development of a GAS vaccine has been impeded by scientific, regulatory, and commercial obstacles(2).

Controlled human infection models (CHIM) are increasingly assuming an important role for vaccine development $(3,4)$. Drawing on the record of historical CHIM studies that included 172 participants (5-7), a modern pharyngitis CHIM in healthy adult volunteers has been proposed as part of a re-energized global effort to accelerate GAS vaccine development(8). Selection of a thoroughly characterized strain is central for development of a GAS CHIM.

A successful CHIM requires that infection and/or symptomatic disease endpoints are reached reliably, safely, and bear sufficient resemblance to a natural state to suggest generalizability. The diverse clinical and microbiological profile of GAS presents challenges for CHIM study design, especially strain selection. There are more than 200 different GAS emm-types. This widely-used classification system is based on one part of the gene encoding a single GAS antigen, the M protein. No other antigen has been as closely studied, and the concept of M protein type-specific immunity has been a cornerstone of GAS research.

GAS is a highly-adapted human pathogen, and the limitations of in vitro assays and animal models have been well described. After more than a century of research, fundamental aspects of pathogenesis and human immune protection against GAS remain unknown. These knowledge gaps are simultaneously an argument for building a CHIM and a source of uncertainty in conceiving its design. A thorough and explicitly stated rationale for strain selection is an important step in minimizing potential harm to participants and maximizing scientific impact. We considered desirable characteristics in selecting an initial strain to establish a GAS pharyngitis CHIM and surveyed available collections for suitable strains, focusing on an emm 75 strain (GAS M75 611024, 'M75') isolated in 2011 from the throat of a 5- 
120

121 In this paper, we present a multifaceted characterisation of the preferred CHIM candidate M75 strain, 122 123 143 isolate.

year-old girl with acute symptomatic pharyngitis in Melbourne (Table 1 and Supplementary Table S1)(9). compared to three others: GAS M12 611025, 'M12', an alternative challenge candidate; M1T1 5448 ('5448'), representative of the M1T1 clone recently responsible for most pharyngitis and invasive disease globally(10); and CDC SS-496, a M1 strain administered to 88 subjects in 1970s pharyngitis CHIM studies ('SS-496’)(5, 7).

\section{Methods}

\section{Bacterial isolates}

M75 611024 and M12 611025 were isolated in 2011 from throat swabs collected from children with acute pharyngitis in Melbourne, Australia, and stored at the Murdoch Children's Research Institute(9). Professor Mark Walker at the University of Queensland supplied 5448(10, 11). The US Centers for Disease Control and Prevention (CDC) Streptococcus Laboratory provided the SS-496 strain, submitted in 1958 from Duke University.

\section{Growth and viability}

For administration to human volunteers, an animal-free medium must sustain sufficient strain growth. A chemically-defined medium was developed ('VR broth') consisting of RPMI-1640 (Gibco) and 2\% (w/v) Veggietone GMO-free soya-peptone (Oxoid). Eight-hour growth assays were done comparing growth of M75 in this medium and in Todd-Hewitt broth (Oxoid) with 1\% (w/v) yeast extract (Bacto)(THY).

Bacteria were grown in $125 \mathrm{ml}$ Erlenmeyer flasks containing $25 \mathrm{ml}$ of VR or THY broth and agitated gently 75 RPM. To simulate manufacturing processes, M75 growth in VR broth was examined after seven days repeated in vitro passage, using frozen cultures of three post-passage clones and the pre-passage parent 
146

147

148

149

150

151

152

153

154

155

156

157

158

159

160

161

162

163

164

165

166

167

168

169

170

171 For the challenge procedure, swab uptake and release of the GAS inoculum should be consistent. We

The HA capsule is a GAS virulence factor, resisting opsonophagocytosis(12). Capsular HA levels were quantified as previously described, using a test kit (Corgenix)(10).

\section{Attachment properties}

Cell culture lines have been used to study GAS adherence(13, 14). We used Detroit 562 (D562) human pharyngeal cells and skin HaCaT cells, simulating natural sites of infection. As previously described, total cell-associated GAS (percentage of original inoculum) and invasiveness (intracellular fraction of total cellassociated GAS) were determined using GAS grown to mid-exponential phase $\left(\mathrm{OD}_{600 \mathrm{~nm}} \sim 0.5\right)$ in VR broth and diluted in $500 \mu \mathrm{l}$ of assay media (MEM with 5\% Fetal Bovine Serum; Gibco) to a multiplicity of infection of 5:1 (GAS:cells)(10). Inoculated trays were centrifuged for five minutes at $200 \mathrm{x} g$ and incubated for one hour at $37^{\circ} \mathrm{C}$ in $5 \% \mathrm{CO}_{2}$, then washed three times with PBS to remove non-adherent bacteria. Cell-associated GAS (adherent plus invasive) were detached using $200 \mu 10.25 \%$ trypsin, lysed with $0.025 \%$ Triton-X (Sigma) in $\mathrm{dH}_{2} \mathrm{O}$, and enumerated by track dilution on horse blood agar (HBA). To measure invasive bacteria cells were washed once after incubation in assay media for one hour then incubated for another hour in media containing $100 \mu \mathrm{g} / \mathrm{ml}$ gentamicin and enumerated as before.

\section{Delivery characteristics}

To assess $\mathrm{M} 75$ viability following storage at $-80^{\circ} \mathrm{C}$, bacteria were grown in VR broth $\left(\mathrm{OD}_{600 \mathrm{~nm}}=0.5\right)$, centrifuged and suspended in broth containing $10 \%$ (v/v) glycerol (Sanofi). Vials containing 10 $, 10^{6}, 10^{7}$, and $10^{8} \mathrm{CFU} / \mathrm{ml}$ were thawed at intervals and immediately tested without washing (mimicking the challenge protocol) for: 1) growth in solid and liquid media; 2) viability by enumeration; and 3) attachment properties, as above.

172 simulated direct oropharyngeal application using four Copan swabs compared to cotton: 
173 FLOQSwab ${ }^{\mathrm{TM}}$ (nylon), Dacron ${ }^{\mathrm{TM}}$ (polyester), and small (S) and large (L) rayon swabs. For uptake, vials of

174 broth were weighed before and after dipping of swabs for 10 seconds. Swab release of GAS was measured 175 by dipping swabs in $1 \mathrm{ml}$ vials containing $1-3 \times 10^{5} \mathrm{CFU}$ of M75 for 10 seconds followed by transferring to $1761 \mathrm{ml}$ of PBS for 10 seconds, then enumerated by spread plate dilutions.

177

\section{Antibiotic susceptibility testing}

Minimum inhibitory concentrations (MIC) were determined by E-test, and double disk diffusion (D-zone test) used to detect inducible clindamycin resistance. Interpretive breakpoints of the Clinical and Laboratory Standards Institute (penicillin, erythromycin, azithromycin, clindamycin, levofloxacin) and European Committee on Antimicrobial Susceptibility Testing (rifampicin) were used(15, 16).

\section{Whole genome sequencing and phylogenetic analyses}

The complete M75 611024 genome sequence was determined using long-read single molecule real-time sequencing on the Pacific Biosciences RS II platform. Filtering of the long reads identified 104,694 reads with an average polymerase read length of $4.1 \mathrm{~kb}$. A single circular assembly was generated using SMRT analysis v2.3.0 (Pacific Biosciences), HGAP v3 and polished using Quiver at an average read depth of 96fold. To aid assembly validation, M75 was also sequenced on an Illumina Next-seq 500 to produce pairedend reads with a read length of 150 bases. The M75 611024 genome sequence has been submitted to GenBank (accession number CP033621). The genomes of M12 611025 and CDC SS-496 were sequenced by Illumina Next-seq 500 with a paired-end read length of 150 bases. Draft genome assemblies were generated using SPAdes v3.12.0. Illumina short reads of M12 611025 (accession number SRR8217179) and CDC SS-496 (SRR8217180) have been submitted to the Short Read Archive (PRJNA504701).

To study M75 genomic stability, three clones were sequenced by Illumina Next-seq 500, 150 bp paired-end reads, after seven days of repeated in vitro passage. These sequences were aligned with the pre-passage parent M75 reference sequence to identify single nucleotide polymorphisms (SNP). 
Phylogenetic analysis of a global dataset of emm 75 isolates was determined by mapping short read 201 sequences of 131 global emm75 genomes from the UK $(n=124), \operatorname{USA}(n=4)$, and France $(n=3)(17-19)$ to 202 the M75 611024 reference genome with BWA MEM (v0.7.16). Single nucleotide polymorphisms (SNPs) 203 with a Phred quality score $\geq 30$ were identified in each isolate using SAMtools pileup with a minimum coverage of 30x. Prophage sequences within M75 611024 were identified using the Phaster server with SNPs located within these prophage excluded as they represent evolutionary confounders. A maximum likelihood phylogenetic tree was built from 1,046 concatenated SNP sites using RAxML v8.2.8 with the general time-reversible model and gamma correction with 100 bootstrap re-samplings to assess phylogenetic support.

Phylogeny of $141 \mathrm{emm} 12$ genomes, including the Illumina reads of M12 611025 and sequences from Australia, USA, Hong Kong and mainland China, was analyzed by mapping to the reference genome HKU16 (strain QMH11M0907901 (GenBank AFRY01000001) from 1,452 vertically inherited SNPs as previously described (20). Illumina reads of the M1 genome sequence CDC SS-496 were mapped to MGAS5005 (GenBank NC_007297) and other M1 reference genomes with phylogeny inferred form 780 vertically inherited SNPs.

\section{Virulence factors and vaccine antigens}

Virulence gene carriage was determined for M75, M12, 5448, and SS-496, by blastN screening assemblies against the virulence factor database (VFDB)(21). Gene presence was defined by an $80 \%$ nucleotide cutoff over $80 \%$ of gene length.

Protection in animal models has been shown for more than twenty-five candidate GAS vaccine protein antigens and several peptide-based antigens(22). For protein antigens, presence was defined by an $80 \%$ nucleotide cut-off over $80 \%$ of gene length. For sequence-constrained peptide-based vaccine epitopes: J8.0 
B-cell (EASRKGLRRDLDASREAKKQVEKA), and common B-T-cell epitopes (KGLRRDLDASREA-

KKQ); a $100 \%$ nucleotide sequence match was taken to define presence, although $100 \%$ homology may not be required to induce production of broadly cross-reactive antibodies and vaccine protection.

\section{Neutrophil killing assay}

Survival of M75, M12, and 5448 incubated with human neutrophils in vitro was assayed as previously described(25). Experiments were performed in triplicate using mid-exponential phase GAS at a multiplicity of infection of 10:1. Differences in neutrophil survival were analysed using 1-way analysis of variance (Graphpad Prism).

\section{Murine invasive model}

Strain virulence was compared in a humanized plasminogen transgenic AlbPLG1 mouse model(26). In separate experiments, groups $(\mathrm{n}=10)$ of AlbPLG1 (+/-) mice were administered subcutaneous doses of

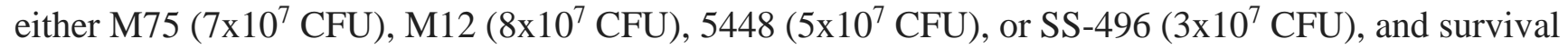
monitored for ten days, as previously described(27, 28).

\section{Ethics statement}

Animal procedures followed the Australian Code for the Care and Use of Animals for Scientific Purposes and were approved by the University of Queensland Animal Ethics Committee (SCMB/140/16/NHMRC)(29). An initial dose-ranging CHIM study has been approved by The Alfred Hospital Ethics Committee (500/17) and is registered at ClinicalTrials.gov (NCT03361163).

\section{Results}

Growth in an animal-free medium

Compared to THY broth, no detrimental effect on growth of M75, M12, and 5448 was observed in the animal-free media (Figure 1A). Eight-hour growth curves for M75 clones tested after seven days repeated in vitro passage were similar to the non-passaged parent (data not shown). 
255

256

257

258

259

260

261

262

263

264

265

266

267

268

269

270

271

272

273

274

275

276

277

278

279

Attachment properties

M75 had the highest adherence to D562 (75\%) and HaCaT (81\%) cells (Figure 1B). M12 (53\%) and 5448

(51\%) were similarly adherent to D562 cells. M12 preferentially adhered to D562 over HaCaT cells

( $p=0.005)$, whereas M75 and M1T1 showed no preference. The affinity of M12 to pharyngeal over skin

cells matches its designation as an A-C pattern strain, associated with throat tissue tropism(30).

Invasiveness of M75 and M12 was low for both cell lines $(\leq 0.45 \%)$. Invasion by 5448 of HaCaT cells (10\%) was greater than for D562 cells $(0.2 \%)$ (Figure 1B).

\section{Capsule production}

M75 produced $74 \mathrm{ng} / \mathrm{ml}$ of HA capsule, compared to M12 which produced $7506 \mathrm{ng} / \mathrm{ml}$ (Figure 1C).

Capsule production by 5448 matched previous findings(31).

\section{Delivery characteristics and viability}

The Dacron ${ }^{\mathrm{TM}}$ swab was considered most suitable for delivery of the challenge inoculum (Supplementary Figure S1). Mean broth uptake by Dacron ${ }^{\mathrm{TM}}(105 \mathrm{mg})$ and Rayon S (108mg) swabs was comparable to cotton $(129 \mathrm{mg})$ and uptake variance was lowest $(7.7 \mathrm{mg})$ for the Dacron ${ }^{\mathrm{TM}}$ swab. Superior release was noted for the Dacron swab with a mean of $1.8 \times 10^{3} \mathrm{CFU}$ of M75 recovered after swab dipping (Figure 2B). Recovery from M75 vials frozen for four months did not fall below $95 \%$ of the original inoculum at $\mathrm{T}=0$. Adherence and invasion were similarly unaffected by storage (data not shown).

Antibiotic susceptibility

M75 was susceptible to all tested antibiotics, while M12 was resistant to macrolides and fluoroquinolones (Table 2). All strains were susceptible to clindamycin, and inducible resistance was not detected.

\section{Whole genome sequencing and phylogenetic analyses}


The complete genome of M75 611024 is comprised of a single chromosome of 1,852,894 bp (Figure 2A).

M75 has the multi-locus sequence type (MLST) ST150 and contains the emm75.0 allele, and mrp24 and enn334 alleles corresponding to the emm-like genes mrp and enn (P. Smeesters, personal communication, July 2018). Three putative prophage sequences were identified in M75 harboring the endonuclease streptodornase 3 (spd3); pyrogenic exotoxins speL and speM; and the endonuclease $s d n$. M75 shared a hypothetical ancestral relationship with a UK emm75 cluster (Figure 2B) yet represents a distinct evolving lineage, suggesting an ancestral relationship to modern day ST150 emm75 clones.

One SNP was found for each of three M75 clones sequenced after seven days repeated in vitro passage, compared to the non-passaged parent strain. Each SNP was intergenic and different, suggestive of random mutations of unlikely functional consequence (data not shown).

M12 611025 belongs to MLST ST36 and carries the emm12.0 allele. It shares a high degree of genome conservation with other emm 12 genome sequences, varying in prophage and integrative conjugative element content relative to the reference genomes HKU16 and MGAS9429 (Figure 2C). Phylogenetic analysis alongside 141 extant emm12 isolates showed an evolutionary relationship with other modern ST36 strains (Figure 2D) including recent scarlet fever outbreak strains(20).

The historical challenge strain SS-496 shares a higher degree of genetic and evolutionary similarity with the ancestral M1 reference strain SF370, relative to the modern M1T1 strains MGAS5005 and 5448 (Figures 2E, 2F)(32). SS-496 contains the historical SF370-like purA to nadC genomic region encoding Streptolysin O.

\section{Virulence factors and vaccine antigens}

M75, M12, and SS-496 carry genes for an array of adhesion and invasion factors common to many emmtypes (Table 3). M75 contains a frameshift mutation in the fibronectin binding protein Sfb1 within the FCT locus. M12 carries the streptococcal superantigen A ( $s s a)$ gene recently reported in scarlet fever-associated 
isolates in China and the UK(33). M12 does not carry the multidrug-resistant integrative conjugative

308 element ICE-emm12 or the ssa-carrying prophage $\Phi H K U . v i r$ linked to the emergence of scarlet fever clades(20). The virulence profile of SS-496 is similar to pre-1980 M1 strains such as SF370, with speH and speI exotoxins and absence of the speA exotoxin typical of modern isolates such as 5448 (Table 2). M75,

M12, SS-496 and 5448 all possess wildtype $\operatorname{cov} R / S$ and $\operatorname{rop} B$ two-component virulence regulators.

High carriage (>60\%) of protein and peptide candidate vaccine antigens was observed for M75 and M12 using a homology-based genome approach (Table 4).

\section{Human neutrophil killing assay}

317 M75 was most susceptible to in vitro killing when incubated with human neutrophils, although killing was 318 observed for all strains (Figure 1D).

\section{Mouse lethal invasive model}

Compared to M12, 5448, and SS-496, M75 was avirulent in the humanized mouse invasive disease model (Figure 1E).

\section{Discussion}

We have described the rationale for selecting M75 for initial use in a new GAS pharyngitis CHIM in healthy adults, including results of diverse pre-clinical studies assessing its fitness for purpose. For context and comparison, we have presented results for three other strains (M12, 5448, SS-496).

M75 is compatible with critical protocol points: reliable growth in an animal-free medium; retains growth and attachment properties after prolonged storage at $-80^{\circ} \mathrm{C}$; consistent delivery using a commercially available swab; and susceptibility to antibiotics used to treat GAS pharyngitis. M75 looks to have an acceptable virulence profile, with capacity to cause pharyngitis and low potential for invasive disease. M75 
cellular invasion. M75 was highly susceptible to in vitro killing by human neutrophils, possibly due to its minimal capsular HA production. In a humanized mouse model of invasive infection, M75 was avirulent whereas M12 and both M1 strains were lethal. Whole genome sequencing placed the strains in the context of epidemiologically-related phylotypes, found broad representation of candidate vaccine antigens, and a relatively restricted array of virulence factor genes in M75.

CHIM strain selection has been guided by varied general and pathogen-specific considerations, all with the goal of safely and reliably reproducing relevant and generalisable asymptomatic (infection/carriage) or symptomatic (disease) study endpoints $(3,4,34)$. Suitable well-characterized strains may already exist, such as the Salmonella Typhi Quailes strain(35). Patients with mild-moderately severe uncomplicated disease may be a source of 'naturally attenuated' new strains. Multiple strains, sometimes from different locations, may be required to represent natural strain diversity and/or enable heterologous re-challenge(36). If mechanisms of severe infection and/or complications are known, the implicated virulence factor(s) may be avoided (e.g. Shiga toxin-producing Escherichia coli(37) and Campylobacter jejuni inducing crossreactive antibodies to GM1 and GQ1b gangliosides(38)). Pathogens may be modified for use, such as the propagation of single sex Schistosoma mansoni cercariae to prevent chronic schistosomiasis(39). For vaccine studies, target antigen(s) must be present in the challenge strain(s). In every instance, strains must be characterized and be compatible with protocols for manufacturing, inoculation, and techniques to measure organism and host responses.

The limitations of this characterization effort are inherent in the rationale for pursuing a GAS CHIM. In vitro assays, genomics, and animal models do not fully capture or predict the dynamic elements and sequelae of human infection by GAS, a highly-adapted and human-restricted pathogen. Even advanced non-human primate models produce a pharyngitis syndrome with important differences from human disease. A single contemporary clone, represented here by 5448, is simultaneously the most common cause in urbanized settings of both the mildest and most severe disease syndromes, with the basis for tissuetropism and bacterial-human genotype-phenotype relationships still relatively obscure. These uncertainties 
362 dictate a cautious approach extending beyond strain selection, including: strain manufacture following 363 principles of Good Manufacturing Practice; initial inclusion of healthy adults only without risk factors for 364 severe GAS disease; a dose-ranging study to establish attack rate and safety; inpatient admission at a trials 365 facility supported by a tertiary hospital; universal antibiotic treatment; outpatient follow-up; and 366 echocardiography at screening and final visits.

368 A generic limitation of CHIM studies is the uncertain degree to which data from healthy adults 369 experiencing a single syndrome (pharyngitis) caused by one strain (M75) can be generalized to other 370 subjects, syndromes, strains, and settings (e.g. children with GAS skin infections due to other emm-types in 371 low- and middle-income countries). While inclusion of other strains and even a skin infection CHIM are 372 conceivable extensions, model findings must be interpreted alongside knowledge derived from more 373 naturalistic studies. For vaccine development, a GAS pharyngitis CHIM has dual scientific and strategic 374 purposes, aiming to serve as a bridge to field trials with a more natural distribution of subjects, syndromes, 375 and strains.

377 With a view to the very high priority given to participant safety and risk minimization, findings from these 378 strain characterization studies reinforce the appropriateness of M75 for initial use in a GAS pharyngitis 379 CHIM. 


\section{Funding}

384 This work is supported by the Australian National Health and Medical Research Council [GNT1099183;

385 GNT1133299 to J.O; GNT1126805 to M.R.D; GNT1102621, GNT1071659, GNT1074899 to M.J.W; and 386 GNT1127077 to A.S]; the Australian Heart Foundation [to A.S]; the Garnett Passe and Rodney Williams 387 Memorial Foundation [to C.L.Y.O]; and the Belgian National Fund for Scientific Research (FNRS) [PDR T.0255.16, CDR J.0019.17 to P.R.S].

Transparency declaration

The lead author affirms that this manuscript is an honest, accurate, and transparent account of the study being reported; that no important aspects of the study have been omitted; and that any discrepancies from the study as planned have been explained. The authors have no financial or other conflicts to declare.

\section{Acknowledgements}

The Controlled Human Infection for Vaccination against Streptococcus pyogenes (CHIVAS) study group includes Andrew Steer, Anneke Grobler (Murdoch Children's Research Institute); Michael Batzloff, Michael Good, and Manisha Pandey (Griffith University); Jonathan Carapetis (Telethon Kids Institute),

Pierre Smeesters (Université Libre de Bruxelles); Claire Waddington (University of Cambridge); Jim Dale (University of Tennessee); Tibor Schuster (McGill University); Allen Cheng (Monash University); Andrew Pollard (Oxford University); James McCarthy (QIMR Berghofer); and Mark Walker (University of Queensland).

Thanks to Dr. Deborah Williamson, Dr. Kate Worthing and staff at the Microbiological Diagnostic Unit Public Health Laboratory, The Peter Doherty Institute for Infection and Immunity, The University of Melbourne. Thanks to Dr. Bernard Beall and staff at the US Centers for Disease Control and Prevention Streptococcus Laboratory. We acknowledge the assistance of the sequencing and pathogen informatics core teams at the Wellcome Trust Sanger Institute and support for the genome sequencing components 
Networks in Vaccines Research and Development, which was co-funded by the Medical Research Council

(MRC) and Biotechnology and Biological Sciences Research Council (BBSRC).

\section{References}

415 1. Walker MJ, Barnett TC, McArthur JD, Cole JN, Gillen CM, Henningham A, Sriprakash KS, Sanderson-Smith ML, Nizet V. 2014. Disease manifestations and pathogenic mechanisms of Group A Streptococcus. Clin Microbiol Rev 27:264-301.

2. Steer AC, Carapetis JR, Dale JB, Fraser JD, Good MF, Guilherme L, Moreland NJ, Mulholland EK, Schodel F, Smeesters PR. 2016. Status of research and development of vaccines for Streptococcus pyogenes. Vaccine 34:2953-8.

3. Roestenberg M, Hoogerwerf MA, Ferreira DM, Mordmuller B, Yazdanbakhsh M. 2018. Experimental infection of human volunteers. Lancet Infect Dis doi:10.1016/S1473-3099(18)301774.

4. Anonymous. 2018. Controlled Human Infection Model Studies: Summary of a workshop held on 6 February 2018. . The Academy of Medical Sciences., Sciences. TAoM,

5. Polly SM, Waldman RH, High P, Wittner MK, Dorfman A. 1975. Protective studies with a group A streptococcal M protein vaccine. II. Challange of volenteers after local immunization in the upper respiratory tract. J Infect Dis 131:217-24.

6. D'Alessandri R, Plotkin G, Kluge RM, Wittner MK, Fox EN, Dorfman A, Waldman RH. 1978. Protective studies with group A streptococcal M protein vaccine. III. Challenge of volunteers after systemic or intranasal immunization with Type 3 or Type 12 group A Streptococcus. J Infect Dis 138:712-8.

7. Fox EN, Waldman RH, Wittner MK, Mauceri AA, Dorfman A. 1973. Protective study with a group A streptococcal M protein vaccine. Infectivity challenge of human volunteers. J Clin Invest 52:1885-92.

8. Osowicki J, Vekemans J, Kaslow DC, Friede MH, Kim JH, Steer AC. 2018. WHO/IVI global stakeholder consultation on group A Streptococcus vaccine development: Report from a meeting held on 12-13 December 2016. Vaccine 36:3397-3405.

9. Dunne EM, Marshall JL, Baker CA, Manning J, Gonis G, Danchin MH, Smeesters PR, Satzke C, Steer AC. 2013. Detection of group a streptococcal pharyngitis by quantitative PCR. BMC Infect Dis 13:312.

10. Hollands A, Pence MA, Timmer AM, Osvath SR, Turnbull L, Whitchurch CB, Walker MJ, Nizet V. 2010. Genetic switch to hypervirulence reduces colonization phenotypes of the globally disseminated group A streptococcus M1T1 clone. J Infect Dis 202:11-9.

11. Chatellier S, Ihendyane N, Kansal RG, Khambaty F, Basma H, Norrby-Teglund A, Low DE, McGeer A, Kotb M. 2000. Genetic relatedness and superantigen expression in group A streptococcus serotype M1 isolates from patients with severe and nonsevere invasive diseases. Infect Immun 68:3523-34.

12. Dale JB, Washburn RG, Marques MB, Wessels MR. 1996. Hyaluronate capsule and surface M protein in resistance to opsonization of group A streptococci. Infect Immun 64:1495-501.

13. Loh JMS, Tsai JC, Proft T. 2017. The ability of Group A streptococcus to adhere to immortalized human skin versus throat cell lines does not reflect their predicted tissue tropism. Clin Microbiol Infect 23:677 e1-677 e3.

14. Ryan PA, Juncosa B. 2016. Group A Streptococcal Adherence. In Ferretti JJ, Stevens DL, Fischetti VA (ed), Streptococcus pyogenes : Basic Biology to Clinical Manifestations, Oklahoma City (OK). 
15. Clinical and Laboratory Standards Institute. 2016. Performance Standards for Antimicrobial Susceptibility Testing: Twenty-sixth Informational Supplement M100-S26, CLSI, Wayne, PA, USA.

16. Anonymous. The European Committee on Antimicrobial Susceptibility Testing. Breakpoint tables for interpretation of MICs and zone diameters. Version 6.0, 2016., http://www.eucast.org.

17. Kapatai G, Coelho J, Platt S, Chalker VJ. 2017. Whole genome sequencing of group A Streptococcus: development and evaluation of an automated pipeline for emmgene typing. PeerJ 5:e3226.

18. Chochua S, Metcalf BJ, Li Z, Rivers J, Mathis S, Jackson D, Gertz RE, Jr., Srinivasan V, Lynfield R, Van Beneden C, McGee L, Beall B. 2017. Population and Whole Genome Sequence Based Characterization of Invasive Group A Streptococci Recovered in the United States during 2015. MBio 8.

19. Rochefort A, Boukthir S, Moullec S, Meygret A, Adnani Y, Lavenier D, Faili A, Kayal S. 2017. Full Sequencing and Genomic Analysis of Three emm75 Group A Streptococcus Strains Recovered in the Course of an Epidemiological Shift in French Brittany. Genome Announc 5.

20. Davies MR, Holden MT, Coupland P, Chen JH, Venturini C, Barnett TC, Zakour NL, Tse H, Dougan G, Yuen KY, Walker MJ. 2015. Emergence of scarlet fever Streptococcus pyogenes emm 12 clones in Hong Kong is associated with toxin acquisition and multidrug resistance. Nat Genet 47:84-7.

21. Chen L, Zheng D, Liu B, Yang J, Jin Q. 2016. VFDB 2016: hierarchical and refined dataset for big data analysis--10 years on. Nucleic Acids Res 44:D694-7.

22. Henningham A, Gillen CM, Walker MJ. 2013. Group a streptococcal vaccine candidates: potential for the development of a human vaccine. Curr Top Microbiol Immunol 368:207-42.

23. Batzloff MR, Hayman WA, Davies MR, Zeng M, Pruksakorn S, Brandt ER, Good MF. 2003. Protection against group A streptococcus by immunization with J8-diphtheria toxoid: contribution of J8- and diphtheria toxoid-specific antibodies to protection. J Infect Dis 187:1598-608.

24. Guilherme L, Fae KC, Higa F, Chaves L, Oshiro SE, Freschi de Barros S, Puschel C, Juliano MA, Tanaka AC, Spina G, Kalil J. 2006. Towards a vaccine against rheumatic fever. Clin Dev Immunol 13:125-32.

25. Buchanan JT, Simpson AJ, Aziz RK, Liu GY, Kristian SA, Kotb M, Feramisco J, Nizet V. 2006. DNase expression allows the pathogen group A Streptococcus to escape killing in neutrophil extracellular traps. Curr Biol 16:396-400.

26. Sun H, Ringdahl U, Homeister JW, Fay WP, Engleberg NC, Yang AY, Rozek LS, Wang X, Sjobring U, Ginsburg D. 2004. Plasminogen is a critical host pathogenicity factor for group A streptococcal infection. Science 305:1283-6.

27. Walker MJ, Hollands A, Sanderson-Smith ML, Cole JN, Kirk JK, Henningham A, McArthur JD, Dinkla K, Aziz RK, Kansal RG, Simpson AJ, Buchanan JT, Chhatwal GS, Kotb M, Nizet V. 2007. DNase Sda1 provides selection pressure for a switch to invasive group A streptococcal infection. Nat Med 13:981-5.

28. Maamary PG, Sanderson-Smith ML, Aziz RK, Hollands A, Cole JN, McKay FC, McArthur JD, Kirk JK, Cork AJ, Keefe RJ, Kansal RG, Sun H, Taylor WL, Chhatwal GS, Ginsburg D, Nizet V, Kotb M, Walker MJ. 2010. Parameters governing invasive disease propensity of non-M1 serotype group A streptococci. J Innate Immun 2:596-606.

29. Anonymous. National Health and Medical Research Council. 2013. Australian code for the care and use of animals for scientific purposes, 8th ed. National Health and Medical Research Council, Canberra, Australia.

30. Bessen DE, Smeesters PR, Beall BW. 2018. Molecular Epidemiology, Ecology, and Evolution of Group A Streptococci. Microbiol Spectr 6.

31. Cole JN, Pence MA, von Kockritz-Blickwede M, Hollands A, Gallo RL, Walker MJ, Nizet V. 2010. M protein and hyaluronic acid capsule are essential for in vivo selection of covRS mutations characteristic of invasive serotype M1T1 group A Streptococcus. MBio 1.

32. Nasser W, Beres SB, Olsen RJ, Dean MA, Rice KA, Long SW, Kristinsson KG, Gottfredsson M, Vuopio J, Raisanen K, Caugant DA, Steinbakk M, Low DE, McGeer A, Darenberg J, Henriques- 
Normark B, Van Beneden CA, Hoffmann S, Musser JM. 2014. Evolutionary pathway to increased virulence and epidemic group A Streptococcus disease derived from 3,615 genome sequences. Proc Natl Acad Sci U S A 111:E1768-76.

33. You Y, Davies MR, Protani M, McIntyre L, Walker MJ, Zhang J. 2018. Scarlet Fever Epidemic in China Caused by Streptococcus pyogenes Serotype M12: Epidemiologic and Molecular Analysis. EBioMedicine 28:128-135.

34. Darton TC, Blohmke CJ, Moorthy VS, Altmann DM, Hayden FG, Clutterbuck EA, Levine MM, Hill AV, Pollard AJ. 2015. Design, recruitment, and microbiological considerations in human challenge studies. Lancet Infect Dis 15:840-51.

35. Waddington CS, Darton TC, Jones C, Haworth K, Peters A, John T, Thompson BA, Kerridge SA, Kingsley RA, Zhou L, Holt KE, Yu LM, Lockhart S, Farrar JJ, Sztein MB, Dougan G, Angus B, Levine MM, Pollard AJ. 2014. An outpatient, ambulant-design, controlled human infection model using escalating doses of Salmonella Typhi challenge delivered in sodium bicarbonate solution. Clin Infect Dis 58:1230-40.

36. Stanisic DI, McCarthy JS, Good MF. 2018. Controlled Human Malaria Infection: Applications, Advances, and Challenges. Infect Immun 86.

37. Harro C, Chakraborty S, Feller A, DeNearing B, Cage A, Ram M, Lundgren A, Svennerholm AM, Bourgeois AL, Walker RI, Sack DA. 2011. Refinement of a human challenge model for evaluation of enterotoxigenic Escherichia coli vaccines. Clin Vaccine Immunol 18:1719-27.

38. Tribble DR, Baqar S, Carmolli MP, Porter C, Pierce KK, Sadigh K, Guerry P, Larsson CJ, Rockabrand D, Ventone CH, Poly F, Lyon CE, Dakdouk S, Fingar A, Gilliland T, Daunais P, Jones E, Rymarchyk S, Huston C, Darsley M, Kirkpatrick BD. 2009. Campylobacter jejuni strain CG8421: a refined model for the study of Campylobacteriosis and evaluation of Campylobacter vaccines in human subjects. Clin Infect Dis 49:1512-9.

39. Janse JJ, Langenberg MCC, Kos-Van Oosterhoud J, Ozir-Fazalalikhan A, Brienen EAT, Winkel BMF, Erkens MAA, van der Beek MT, van Lieshout L, Smits HH, Webster BL, Zandvliet ML, Verbeek R, Westra IM, Meij P, Visser LG, van Diepen A, Hokke CH, Yazdanbakhsh M, Roestenberg M. 2018. Establishing the Production of Male Schistosoma mansoni Cercariae for a Controlled Human Infection Model. J Infect Dis 218:1142-1146.

40. O'Loughlin RE, Roberson A, Cieslak PR, Lynfield R, Gershman K, Craig A, Albanese BA, Farley MM, Barrett NL, Spina NL, Beall B, Harrison LH, Reingold A, Van Beneden C. 2007. The epidemiology of invasive group A streptococcal infection and potential vaccine implications: United States, 2000-2004. Clin Infect Dis 45:853-62.

41. Nelson GE, Pondo T, Toews KA, Farley MM, Lindegren ML, Lynfield R, Aragon D, Zansky SM, Watt JP, Cieslak PR, Angeles K, Harrison LH, Petit S, Beall B, Van Beneden CA. 2016. Epidemiology of Invasive Group A Streptococcal Infections in the United States, 2005-2012. Clin Infect Dis 63:478-86.

42. Alikhan NF, Petty NK, Ben Zakour NL, Beatson SA. 2011. BLAST Ring Image Generator (BRIG): simple prokaryote genome comparisons. BMC Genomics 12:402. 


\section{Tables}

\section{Table 1. Preferred strain characteristics for a controlled-human infection model of GAS pharyngitis*}

\begin{tabular}{lll} 
Desirable strain characteristics & Rationale \\
\hline
\end{tabular}

A definite but uncommon contemporary cause of symptomatic pharyngitis

Should cause skin infection

An uncommon cause of invasive GAS disease and immunological sequelae

Should have predictable and limited virulence, and be suitable for use in anima models

Should have limited antibiotic resistance

The challenge strain should possess a wide array of candidate vaccine antigen
Pharyngitis is the critical early target for GAS vaccine development Historical CHIM studies offer a template for a reliable and safe protocol. GAS pharyngitis is most common in childhood and adolescence, suggesting previous exposure and immune memory could prevent experimentallyinduced disease in adult volunteers.

Common GAS skin infections (e.g. impetigo) will also be important in initia vaccine field trials. Ideally, the pharyngitis CHIM strain(s) should also be suitable for use in a potential future human model of cutaneous GAS infection.

GAS pharyngitis can lead to locally-invasive infectious complications (e.g. retropharyngeal abscess), severe invasive infection (e.g. streptococcal toxicshock syndrome, STSS), acute rheumatic fever and glomerulonephritis.

Whole genome sequencing, in vitro assays and animal models may inform understanding of a GAS strain's relative virulence, although none fully predict human disease patterns.

Ideally, the challenge strain should be eradicated from the pharynx by antibiotic treatment. Resistance to penicillin has not been documented in GAS, however it does not reliably eradicate GAS from the pharynx. Resistance is variably observed to other drugs.

For greatest impact, a GAS pharyngitis CHIM should be suitable for early use as a preliminary testing ground for vaccines.
- $\quad$ From a child with symptomatic GAS pharyngitis in Melbourne, 2011

- Pre-existing immunity in adults is unknown (no correlate of protection)

- $\leq 5 \%$ of strains in most recent pharyngitis studies are emm75

- E pattern 'generalist' (throat and skin infections)

- Cluster E6 - linked phylogenetically to D pattern skin isolates

- $\quad \leq 5 \%$ of isolates in recent reports of invasive GAS are emm75; from 2000 2016, 403/17,002 (2.4\%) typeable invasive isolates reported to the US CDC's Active Bacterial Core surveillance were emm 75 (Chris A. Van Beneden, Personal Communication, September $\left.11^{\text {th }}, 2018\right)(40,41)$

- $\quad$ emm75 strains rarely associated with ARF/RHD or APSGN (1)

- $\quad \operatorname{covR} / \mathrm{S}$ virulence regulator wildtype (non-mutant)

- Does not bind plasminogen and fibrinogen

- $\quad$ emm75 strains have been used in animal nasopharyngitis and invasive disease models

- $\quad$ See text

- See text

* See web-only Supplementary Table S1 for a detailed and referenced version of this table

ARF: acute rheumatic fever; APSGN: acute post-streptococcal glomerulonephritis; CDC: Centers for Disease Control; CHIM: controlled human infection model; GAS: group A

Streptococcus; RHD: rheumatic heart disease 
Table 2. Antibiotic susceptibility of contemporary group A streptococcal strains M75 611024, M12 611025, and M1T1 5448

\begin{tabular}{|c|c|c|c|c|c|c|}
\hline \multirow{2}{*}{ Antibiotic } & \multicolumn{3}{|c|}{ Breakpoints $^{\#}(\mathrm{mg} / \mathrm{L})$} & \multicolumn{3}{|c|}{ E-test MIC (mg/L) } \\
\hline & $\mathbf{S}$ & I & $\mathbf{R}$ & M75 & M12 & 5448 \\
\hline Penicillin & $\leq 0.12$ & - & - & 0.012 & 0.016 & 0.012 \\
\hline Erythromycin & $\leq 0.25$ & 0.5 & $\geq 1$ & 0.094 & 16 & 0.125 \\
\hline Clindamycin & $\leq 0.25$ & 0.5 & $\geq 1$ & $0.125^{*}$ & $0.125^{*}$ & $0.125^{*}$ \\
\hline Azithromycin & $\leq 0.5$ & 1 & $\geq 2$ & 1 & 64 & 1.5 \\
\hline Levofloxacin & $\leq 2$ & 4 & $\geq 8$ & 0.5 & 4 & 0.5 \\
\hline Rifampicin & $\leq 0.06$ & & $>0.5$ & 0.064 & 0.064 & 0.125 \\
\hline
\end{tabular}

* Inducible clindamycin resistance (D test) not detected

\# All CLSI breakpoints except rifampicin (EUCAST)

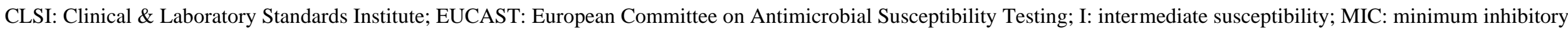
concentration; R: resistant; S: susceptible 
Table 3. Group A Streptococcus virulence factor genomic screen

\begin{tabular}{|c|c|c|c|c|c|}
\hline gene & function & M75 611024 & M12 611025 & M1T1 5448 & CDC SS-496 \\
\hline$c f a / c f b$ & CAMP factor & $\checkmark$ & $\checkmark$ & $\checkmark$ & $\checkmark$ \\
\hline tee $($ сра) & T-pilus antigen & & $\checkmark$ & $\checkmark$ & $\checkmark$ \\
\hline срpA & putative $\mathrm{C} 3$-degrading proteinase & $\checkmark$ & $\checkmark$ & $\checkmark$ & $\checkmark$ \\
\hline emm & M-protein & $\checkmark$ & $\checkmark$ & $\checkmark$ & $\checkmark$ \\
\hline endos & endo-beta-N-acetylglucosaminidase $\mathrm{F} 2$ precursor & $\checkmark$ & $\checkmark$ & $\checkmark$ & $\checkmark$ \\
\hline$f b p 54$ & fibrinogen-binding protein & $\checkmark$ & $\checkmark$ & $\checkmark$ & $\checkmark$ \\
\hline$f c t A$ & major pilin Ap1 (FctA) & & & $\checkmark$ & $\checkmark$ \\
\hline$f c t B$ & minor pilin Ap2 (FctB) & & & $\checkmark$ & $\checkmark$ \\
\hline grab & protein G-related alpha $2 \mathrm{M}$-binding protein & & $\checkmark$ & $\checkmark$ & $\checkmark$ \\
\hline hasA & hyaluronate synthase capsule & $\checkmark$ & $\checkmark$ & $\checkmark$ & $\checkmark$ \\
\hline has $B$ & UDP-glucose 6-dehydrogenase capsule & $\checkmark$ & $\checkmark$ & $\checkmark$ & $\checkmark$ \\
\hline hasC & putative UDP-glucose pyrophosphorylase & $\checkmark$ & $\checkmark$ & $\checkmark$ & $\checkmark$ \\
\hline$h t r A / \operatorname{deg} P$ & serine protease & $\checkmark$ & $\checkmark$ & $\checkmark$ & $\checkmark$ \\
\hline htsA & putative $\mathrm{ABC}$ transporter periplasmic binding protein & $\checkmark$ & $\checkmark$ & $\checkmark$ & $\checkmark$ \\
\hline htsB & putative $\mathrm{ABC}$ transporter permease & $\checkmark$ & $\checkmark$ & $\checkmark$ & $\checkmark$ \\
\hline htsC & putative $\mathrm{ABC}$ transporter $\mathrm{ATP}$-binding protein & $\checkmark$ & $\checkmark$ & $\checkmark$ & $\checkmark$ \\
\hline hyl & hyaluronoglucosaminidase & $\checkmark$ & $\checkmark$ & $\checkmark$ & $\checkmark$ \\
\hline hylA & hyaluronate lyase precursor & & $\checkmark$ & $\checkmark$ & $\checkmark$ \\
\hline hylP & hyaluronoglucosaminidase & $\checkmark$ & $\checkmark$ & $\checkmark$ & $\checkmark$ \\
\hline ideS/mac & IgG-degrading protease & $\checkmark$ & $\checkmark$ & $\checkmark$ & $\checkmark$ \\
\hline lepA & signal peptidase I & & & $\checkmark$ & $\checkmark$ \\
\hline $\operatorname{lmb}$ & laminin binding protein & $\checkmark$ & $\checkmark$ & $\checkmark$ & $\checkmark$ \\
\hline$m f / s p d$ & deoxyribonuclease & $\checkmark$ & $\checkmark$ & $\checkmark$ & $\checkmark$ \\
\hline$m f 3$ & deoxyribonuclease & $\checkmark$ & . & $\checkmark$ & $\checkmark$ \\
\hline plr/gapA & glyceraldehyde-3-phosphate dehydrogenase & $\checkmark$ & $\checkmark$ & $\checkmark$ & $\checkmark$ \\
\hline $\operatorname{prtF2}$ & collagen adhesion protein & & $\checkmark$ & & \\
\hline psaA & manganese-binding protein & $\checkmark$ & $\checkmark$ & $\checkmark$ & $\checkmark$ \\
\hline $\operatorname{sag} A$ & streptolysin S precursor & $\checkmark$ & $\checkmark$ & $\checkmark$ & $\checkmark$ \\
\hline sclA & collagen-like surface protein A & $\checkmark$ & $\checkmark$ & $\checkmark$ & $\checkmark$ \\
\hline$s c l B$ & putative collagen-like protein & & & $\checkmark$ & $\checkmark$ \\
\hline $\operatorname{scp} A$ & C5A peptidase precursor & $\checkmark$ & $\checkmark$ & $\checkmark$ & $\checkmark$ \\
\hline$s d a$ & phage-encoded streptodornase Sda & & $\checkmark$ & $\checkmark$ & \\
\hline$s d n$ & phage-encoded endonuclease Sdn & $\checkmark$ & & & \\
\hline sfbII/sof & fibronectin-binding protein & $\checkmark$ & $\checkmark$ & & \\
\hline$s f b X$ & fibronectin-binding protein & $\checkmark$ & $\checkmark$ & & \\
\hline $\operatorname{shp}$ & hypothetical protein & $\checkmark$ & $\checkmark$ & $\checkmark$ & $\checkmark$ \\
\hline$s h r$ & Fe3+-siderophore transporter & $\checkmark$ & $\checkmark$ & $\checkmark$ & $\checkmark$ \\
\hline sic & streptococcal inhibitor of complement & & & $\checkmark$ & \\
\hline ska & streptokinase precursor & $\checkmark$ & $\checkmark$ & $\checkmark$ & $\checkmark$ \\
\hline slo & streptolysin O & $\checkmark$ & $\checkmark$ & $\checkmark$ & $\checkmark$ \\
\hline smeZ & enterotoxin & $\checkmark$ & $\checkmark$ & $\checkmark$ & $\checkmark$ \\
\hline speB & cysteine protease & $\checkmark$ & $\checkmark$ & $\checkmark$ & $\checkmark$ \\
\hline speA & exotoxin A & & & $\checkmark$ & \\
\hline speG & exotoxin $\mathrm{G}$ & $\checkmark$ & $\checkmark$ & $\checkmark$ & $\checkmark$ \\
\hline speH & exotoxin $\mathrm{H}$ & & $\checkmark$ & & $\checkmark$ \\
\hline speI & exotoxin I & & $\checkmark$ & & $\checkmark$ \\
\hline speJ & exotoxin $\mathrm{J}$ & & & $\checkmark$ & $\checkmark$ \\
\hline speL & exotoxin L & $\checkmark$ & & & \\
\hline speM & exotoxin $\mathrm{M}$ & $\checkmark$ & & & \\
\hline spyA & C3 family ADP-ribosyltransferase & $\checkmark$ & $\checkmark$ & $\checkmark$ & $\checkmark$ \\
\hline $\operatorname{srtCl}$ & sortase & & & $\checkmark$ & $\checkmark$ \\
\hline ssa & streptococcal superantigen A & & $\checkmark$ & & \\
\hline tig/ropA & trigger factor & $\checkmark$ & $\checkmark$ & $\checkmark$ & $\checkmark$ \\
\hline
\end{tabular}


Table 4. Group A Streptococcus candidate vaccine antigen genomic screen*

\begin{tabular}{|c|c|c|c|c|c|}
\hline gene/antigen & gene identifier $^{+}$ & function & $\begin{array}{c}\text { M75 } \\
611024 \\
\end{array}$ & $\begin{array}{c}\text { M12 } \\
611025 \\
\end{array}$ & $\begin{array}{c}\text { M1T1 } \\
5448 \\
\end{array}$ \\
\hline & & M-protein: N-terminal (30-valent vaccine) & $\checkmark$ & $\checkmark$ & $\checkmark$ \\
\hline & & M-protein: C-terminal (J8.0) & & $\checkmark$ & $\checkmark$ \\
\hline & & $\begin{array}{l}\text { M-protein: C-terminal (StreptInCor T-cell } \\
\text { epitope) } \\
\text { M-protein: C-terminal (StreptInCor B-cell } \\
\text { epitope) }\end{array}$ & & & \\
\hline & & $\begin{array}{l}\text { M-protein: C-terminal (StreptInCor common } \\
\text { epitope) }\end{array}$ & & $\checkmark$ & \\
\hline adi & MGAS5005_spy1275 & Arginine deaminase & $\checkmark$ & $\checkmark$ & $\checkmark$ \\
\hline fbaA & MGAS5005_spy1714 & Fibronectin-binding protein A & & & \\
\hline fbp54 & AAA57236 & Fibronectin-binding protein 54 & $\checkmark$ & $\checkmark$ & $\checkmark$ \\
\hline oppA & M5005_spy0249 & Oligopeptide-binding protein & $\checkmark$ & $\checkmark$ & $\checkmark$ \\
\hline GAC & MGAS5005"\# & Group A carbohydrate & $\checkmark$ & $\checkmark$ & $\checkmark$ \\
\hline pulA & SF370_spy1972 & Putative pullulanase & $\checkmark$ & $\checkmark$ & $\checkmark$ \\
\hline $\mathrm{r} 28$ & AF091393 & Rib-like cell wall protein & & & \\
\hline $\operatorname{scpA}$ & MGAS5005_spy1715 & C5a peptidase & $\checkmark$ & $\checkmark$ & $\checkmark$ \\
\hline sfbI & X67947 & Streptococcal fibronectin binding protein I & & & \\
\hline sfbII/sof & X83303 & Serum opacity factor & $\checkmark$ & & \\
\hline $\operatorname{shr}$ & SPY1530 & Streptococcal hemoprotein receptor & $\checkmark$ & $\checkmark$ & $\checkmark$ \\
\hline $\operatorname{sib} 35$ & AB254157 & $\begin{array}{l}\text { Streptococcal immunoglobulin-binding } \\
\text { protein } 35\end{array}$ & $\checkmark$ & $\checkmark$ & $\checkmark$ \\
\hline slo & M5005_spy0124 & Streptolysin O & $\checkmark$ & $\checkmark$ & $\checkmark$ \\
\hline spa & MGAS8232_spyM18_2046 & Streptococcal protective antigen & & & \\
\hline speA & X03929 & Streptococcal pyrogenic exotoxin A & & & $\checkmark$ \\
\hline speB & M5005_spy1735 & Cysteine protease & $\checkmark$ & $\checkmark$ & $\checkmark$ \\
\hline speC & SF370_spy0711 & Streptococcal pyrogenic exotoxin C & & & \\
\hline spy0651 & MGAS5005_spy0651 & Cell surface protein & $\checkmark$ & $\checkmark$ & $\checkmark$ \\
\hline spy0762 & MGAS5005_spy0762 & Hypothetical membrane associated protein & $\checkmark$ & $\checkmark$ & $\checkmark$ \\
\hline spy0942 & MGAS5005_spy0942 & Nucleoside-binding protein & $\checkmark$ & $\checkmark$ & $\checkmark$ \\
\hline spyAD & MGAS5005_spy0229 & Adhesin and division protein & $\checkmark$ & $\checkmark$ & $\checkmark$ \\
\hline spyCEP & MGAS5005_spy0341 & IL-8 serine protease & $\checkmark$ & $\checkmark$ & $\checkmark$ \\
\hline sse & SF370_spy1407 & Serine esterase & & $\checkmark$ & $\checkmark$ \\
\hline tee & MGAS5005_spy0109 & T-antigen & $\checkmark$ & $\checkmark$ & $\checkmark$ \\
\hline tif & SF370_spy1612 & Trigger factor & $\checkmark$ & $\checkmark$ & $\checkmark$ \\
\hline
\end{tabular}

* BLAST analyses at a homology level of $80 \%$ for protein antigens and $100 \%$ for peptide-derived sequences.

+ Nucleotide gene sequences derived from completely sequenced genomes or listed GenBank identifiers. Accession numbers for genome sequences; MGAS5005 (CP000017), SF370 (AE004092) and MGAS8232 (AE009949).

\# GAC operon ( 14.2 kb) refers to MGAS5005 genome coordinates 604873 to 619151. 


\section{Figure legends}

Figure 1. In vitro characterisation of contemporary candidate strains for human challenge

A) Growth kinetics of candidate strains in RPMI-1640 supplemented with 2\% Veggietone (filled) and Todd-Hewitt broth with $1 \%$ yeast extract (open). Means and standard deviation (SD) are representative of three separate experiments done in triplicate. B) Strain attachment and cellular invasion. Means and SD are from three separate experiments with triplicate wells. C) Capsular hyaluronic acid quantification. Mean and SD derived from a single experiment. D) Resistance of M75, M12, and 5448 to killing by human neutrophils, means and SD are from three separate experiments using different blood donors, with seven biological replicates. E) Strain lethality in a humanized plasminogen transgenic AlbPLG1 murine invasive disease model ( $\mathrm{n}=10$ for each strain).

Figure 2. Comparative genomics of M75 611024, M12 611025, M1T1 5448, and M1 CDC SS-496

(A) Circular schematic of GAS M75 611024 showing: GC plot (inner ring) with GC content above the genome average (black) and below (grey); predicted prophage sequences shown in red with associated prophage virulence determinants annotated; and relative position of predicted coding sequences on the forward strand (blue) and reverse strand (gold). (B) Unrooted maximum likelihood tree of $131 \mathrm{emm} 75$ strains from UK, US and France based on 1,046 SNPs relative to the M75 611024 reference genome. Tips of the tree are colour coded based on country of isolation. Location of genomes corresponding to M75 611024 and the completely sequenced emm75 strains from France STAB090229 (CP020027); STAB120304 (CP020082) and STAB14018 (CP014542) are annotated. (C) Comparative BlastN analysis of M12 611025 (blue ring) and MGAS9429 (purple ring) relative to the emm12 reference genome HKU16 (inner black circle). HKU16 GC content and GC skew is indicated in the inner ring while annotated around the outside is the genomic position of known HKU16 mobile genetic elements. (D) Maximum likelihood phylogenetic relationship of strain 611025 with 141 emm12 S. pyogenes from other geographical regions based on 1,452 SNP sites from the core genome of the HKU16 reference genome. Tips of the tree are colour coded based on country of isolation of each isolate. Genomes from completely sequenced emm12 strains MGAS9429 (CP000259), MGAS2096 (CP000261) and HKU16 (QMH11M0907901; AFRY01000001) are annotated. (E) Comparative BlastN analysis of CDC SS-496 and other GAS M1 reference genomes AP1, 5448 and MGAS5005 relative to the SF370 M1 GAS reference genome (inner black circle). (F) Mid-point rooted maximum likelihood phylogenetic relationship of M1 GAS reference genomes based on 780 SNP sites. Tips of the tree are annotated by strain name and colour coded by ring colour from (E) and annotated by strain name. Genomes belonging to SF370-like and MGAS5005-like lineages(32) are clustered by grey shading. Comparative BlastN analyses were generated using BRIG(42). 